\section{Vena-Cava-Filter ohne Benefit?}

\author{
Wie Beobachtungsstudien zeigen, wurden in den letzten \\ 3 Jahrzehnten nach akuten venösen Thromboembolien \\ zusätzlich zu einer Antikoagulation vermehrt Vena-Cava-Filter \\ implantiert. Allerdings ist deren Nutzen-Risiko-Verhältnis bisher \\ unklar. P. Mismetti et al. sind diesem nun nachgegangen. \\ JAMA 2015; 313: 1627-1635
}

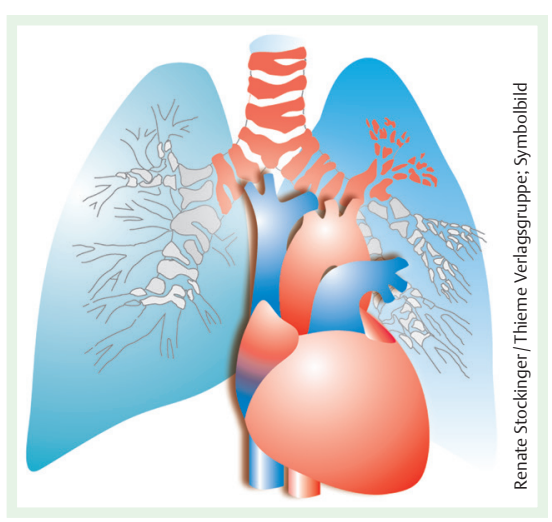

Eingang in die Studie fanden konsekutive Patienten, die aufgrund einer akuten, symptomatischen Lungenembolie als Folge einer akuten Venenthrombose der unteren Extremität stationär behandelt wurden. Die Patienten wurden randomisiert auf 2 Gruppen aufgeteilt und erhielten entweder einen entfernbaren VenaCava-Filter in Kombination mit einer Antikoagulation oder nur eine Antikoagulation. Diese erfolgte für mindestens $6 \mathrm{Mo}-$ nate, anschließend lag das weitere Prozedere im Ermessen des behandelnden Arztes. Die Entfernung des Cava-Filters war 3 Monate nach der Implantation vorgesehen. Primärer Endpunkt der Studie waren symptomatische Rezidiv-Lungenembolien nach 3 Monaten, sekundäre Endpunkte waren Rezidiv-Lungenembolien nach 6 Monaten, symptomatische tiefe Venenthrombosen, Blutungen, Filterkomplikationen sowie die Mortalität nach 3 und 6 Monaten.

\section{Einsatz des Vena-Cava-Filters}

Die Interventionsgruppe bestand aus 200 Patienten, die Kontrollgruppe aus 199. Von den 200 Patienten konnte bei 193 der
Cava-Filter erfolgreich implantiert werden, bei 153 von 164 dafür vorgesehenen Patienten konnte dieser auch wie geplant wieder erfolgreich entfernt werden. In der Kontrollgruppe wurde in der Folge $6 \mathrm{~Pa}-$ tienten ein Filter implantiert.

Nach 3 Monaten hatten in der Cava-Filter-Gruppe 6 Patienten eine Rezidiv-Lungenembolie erlitten (3,0\%), alle endeten tödlich. In der Kontrollgruppe war es zu 3 Rezidiven gekommen (1,5\%), davon 2 mit tödlichem Ausgang. Dies entsprach einem relativen Risiko von 2,0 mit dem Cava-Filter gegenüber der alleinigen Antikoagulation. Im Intervall zwischen 3 und 6 Monaten kam es in beiden Gruppen $\mathrm{zu}$ einer weiteren Lungenembolie. Im Hinblick auf tiefe Venenthrombosen, Blutungen sowie der Gesamtmortalität nach 3 und 6 Monaten fand sich kein deutlicher Unterschied zwischen beiden Gruppen. Bei 3 Patienten kam es zu einer Thrombose des Filters.

\section{Fazit}

Bei hospitalisierten Patienten mit schwerer Lungenembolie konnte die Implantation eines Vena-Cava-Filters in Kombination mit einer Antikoagulation gegenüber einer alleinigen Antikoagulation das Risiko symptomatischer Rezidiv-Lungenembolien innerhalb von 3 Monaten nicht senken. Aufgrund dieser Ergebnisse lässt sich nach Meinung der Autoren der Einsatz des Filters bei Patienten, die antikoaguliert werden können, nicht befürworten.

Dr. Johannes Weiß, Bad Kissingen
Ausschreibung

\section{Karin-Nolte- Wissenschafts-Preis}

Für hervorragende wissenschaftliche Arbeiten auf dem Gebiet der Inneren Medizin verleiht die Saarländisch-Pfälzische Internistengesellschaft (SPIG) seit 1972 den Karin-Nolte-Wissenschaftspreis. Der Preis ist mit einem Preisgeld von $5000 €$ verbunden. Er wird alljährlich anlässlich der Jahrestagung der SPIG verliehen (21. bis 23. April 2016 in Neustadt/Weinstraße). Um den Preis können sich Ärzte bis zum 40. Lebensjahr mit Arbeiten bewerben, die ein wissenschaftliches Thema aus dem Gebiet der Inneren Medizin behandeln. Die Arbeiten dürfen noch nicht veröffentlicht, von anderer Seite mit einem Preis ausgezeichnet oder zu einem anderen Preiswettbewerb eingereicht worden sein. Sie sind in 4-facher Ausfertigung anonym und mit einem Kennwort versehen jeweils bis zum 15. November 2015 (Datum des Poststempels) beim Schriftführer der Gesellschaft, Prof. Dr. M. Uppenkamp, Medizinische Klinik A, Bremserstraße 79 in 67063 Ludwigshafen, einzureichen. Für in englischer Sprache verfasste Arbeiten sind ein deutscher Untertitel und eine deutsche Zusammenfassung erforderlich.In einem verschlossenen Kuvert mit gleichem Kennwort sind Name, Klinik und Adresse des Autors bzw. der Autoren beizufügen. Weitere Informationen zur Ausschreibung finden Sie online unter www.spig.org.

Nach einer Mitteilung der SPIG, Ludwigshafen

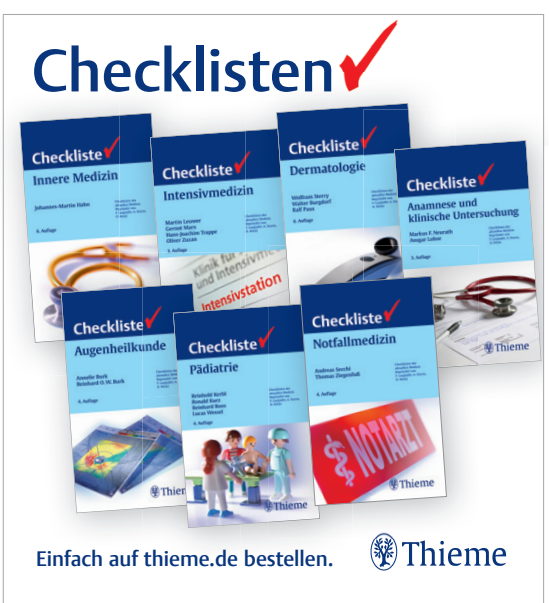

